3. Глинка Н.Л. Общая химия. 24-е изд. - Л.: Химия, 1985. - 702 c.

4. Грег С., Синг К. Адсорбция, удельная пверхность, пористость. - М.: Мир, 1984. - 310 с.

5. Очистка и регенерация нерафинированных растительных масел / Ф.Я. Рудик [и др.] // Известия Тимирязевской сельскохзяйственной академии. - 2019. - № 1. - С. 113-126.

Рудик Феликс Яковлевич, $\partial-p$ техн. наук, проф. кафедры «Технологии продуктов питания», Саратовский государственный аграрный университет имени Н.И. Вавилова. Россия.
Моргунова Наталья Львовна, канд. с.-х. наук, доиент, доцент кафедры «Технологии продуктов питания», Саратовский государственный аграрный университет имени Н.И. Вавилова. Россия.

Сундуков Евгений Александрович, магистрант, Саратовский государственный аграрный университет имени Н.И. Вавилова. Россия.

410005, г. Саратов, ул. Соколовая, 335.

Тел.: (8452) 69-25-32.

Ключевые слова: ультразвук; масло; адсорбциия; очистка; массопередача.

\title{
FACTOR RELATIONSHIP OF THE PARAMETERS OF THE HARMONIC OSCILLATORY SYSTEM WHEN CLEANING THE FILTRATION SURFACE OF THE PLANT FOR VEGETABLE OIL REGENERATION
}

Rudik Phelix Yakovlevich, Doctor of Technical Sciences, Professor of the chair "Food Technology", Saratov State Agrarian University named after N.I. Vavilov. Russia.

Morgunova Natalya Lvovna, Candidate of Agricultural Sciences, Associate Professor of the chair "Food Technology", Saratov State Agrarian University named after N.I. Vavilov. Russia.

Sundukov Evgeniy Aleksandrovich, Magistrandt, Saratov State Agrarian University named after N.I. Vavilov. Russia.

Keywords: ultrasound; oil; adsorption; cleaning; mass transfer.
When processing oilseeds, technologies are needed to obtain valuable vegetable oils by pressing with a long shelf life. Existing technologies for cleaning vegetable oils in small enterprises do not allow cleaning the oil from all undesirable substances and therefore the oils have a short shelf life, quickly oxidize and lose their presentation. The article theoretically substantiates the design parameters of a directional ultrasonic and mechanical oscillatory system for cleaning the filtration surface of the installation from accumulated solid particles in the oil.

\section{МЕТОДИКА ОПРЕДЕЛЕНИЯ ЭКСТРЕМАЛЬНЫХ ЗНАЧЕНИЙ ФУНКЦИОНАЛЬНЫХ ПАРАМЕТРОВ ДВИГАТЕЛЕЙ ПО РЕЗУЛЬТАТАМ ЭКСПЕРИМЕНТАЛЬНЫХ ИССЛЕДОВАНИЙ}

\author{
САВЕЛЬЕВ Анатолий Петрович, Национальный исследовательский Мордовский государственный \\ университет им. Н.П. Огарева
}

ШКРАБАК Владимир Степанович, Санкт-Петербургский государственный аграрный университет

Представлена методика определения экстремальных значений функциональных параметров двигателей при эксплуатационных исследованиях машинно-тракторных агрегатов путем интегрирования функиии двух переменньх.

Введение. Функциональные параметры двигателей при работе машинно-тракторных агрегатов в условиях реальной эксплуатации заметно отличаются от таковых при статистических стендовых испытаниях. Для повышения достоверности диагностирования двигателей по функциональным параметрам приходится прибегать к диагностированию в динамических режимах [1-4].

Максимальная информативность при диагностировании достигается в режиме максимальной эффективной мощности, расхода топлива или удельного расхода топлива. Экстремальные значения эффективной мощности, частоты вращения вала двигателя также необходимы для определения максимальной производительности машинно-тракторных агрегатов. Экстремальные значения функциональных параметров двигателей при неустановившейся нагрузке теоретически определяются с использованием методов функционального преобразования случайных величин и теории механических цепей [1].

Для экспериментального подтверждения теоретических предпосылок исследования проводятся в области максимальной эффективной мощности. Провести исследования в области экстремальных значений невозможно как при стендовых, так и при эксплуатационных испытаниях. В этом случае измерения поводятся как минимум в трех точках в области экстремума, а сам экстремум определяется расчетным путем.

Методика исследований: интерполирование функции двух переменных, регрессионного анализа.

Результаты исследований. При рассмотрении зависимости функциональных параметров двигателей при работе машинно-тракторных агрегатов в функции коэффициента вариации внешней нагрузки $\left(v_{\mathrm{m}}\right)$ получим зависимость второго порядка. Как уже было нами отмечено экспериментальные исследования проводятся в области экстремума функционального параметра, при этом изменяется уровень загрузки двигателя $\lambda_{\text {м. }}$ В этом случае зависимость математического ожидания функционального параметра от $\lambda_{м}$ и $v_{м}$ будет представлять поверхность. С достаточной тонностью приближения можно сказать, что значение 
математических ожиданий функционального параметра располагается по параболической поверхности, или поверхности второго порядка (см. рисунок).

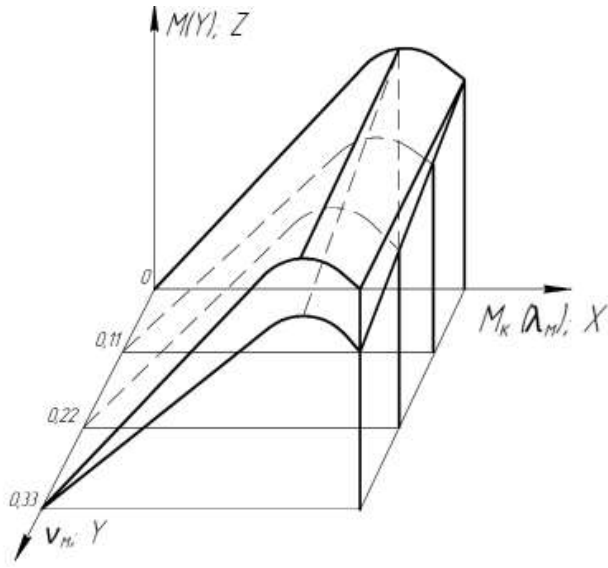

Совокупность математических ожиданий функиионального параметра двигателя при работе в условиях неустановивщейся нагрузки

Выполним математическое описание этой поверхности путем интерполирования поверхностей второго порядка. Функцию $z=f(x, y)$ (см. рисунок) зададим системой равноотстоящих точек $\left.\left(x_{i}, y_{j}\right), i, j=0,1,2 \ldots\right)$, где $x_{i}=x_{0}+i h, y_{j}=y_{0}+j k$, где $h=\Delta x_{i}=$ const, $k=\Delta y_{j}=$ const.

Функцию $z=f(x, y)$ можно представить в виде таблицы с двумя входами (см. таблицу).

Значения функции z в зависимости от параметров $x$ и $y$

\begin{tabular}{|c|c|c|c|c|c|}
\hline & $x_{0}$ & $x_{1}$ & $x_{2}$ & & $x_{m}$ \\
\hline$y_{0}$ & $z_{00}$ & $z_{10}$ & $z_{20}$ & & $z_{m 0}$ \\
\hline$y_{1}$ & $z_{01}$ & $z_{11}$ & $z_{21}$ & & \\
\hline$y_{2}$ & $z_{02}$ & $z_{12}$ & $z_{22}$ & & \\
\hline ... & $\ldots$ & $\ldots$ & $\ldots$ & $\ldots$ & $\ldots$ \\
\hline$y_{n}$ & $z_{0 n}$ & $z_{1 n}$ & $z_{2 n}$ & $\ldots$ & $z_{m n}$ \\
\hline
\end{tabular}

Интерполирование функции двух переменных $z=f(x, y)$, т.е. нахождение ее нетабличных значений, можно последовательно осуществить по каждому переменному $x$ и $y$. Для функции $z=f(x, y)$, представленной в виде двойной таблицы $\left[z_{i}, j\right]$, можно найти частные конечные разности

$$
\Delta_{x} z_{i j}=z_{i+1, j}-z_{i j} \text { и } \Delta_{y} z_{i j}=z_{i+1, j}-z_{i j} \text {. }
$$

При повторном применении этих операций получим двойные разности высших порядков:

$$
\begin{gathered}
\Delta^{m+n} z_{i j}=\Delta_{x}^{m+n} m_{y} n z_{i j}=\Delta_{x}^{m} m\left(\Delta_{y}^{n} n z_{i j}\right)= \\
=\Delta_{y}^{n}\left(\Delta_{x}^{m} m z_{i j}\right),
\end{gathered}
$$

где $\Delta^{0+0} z_{i j}=z_{i j}$. Например:

$$
\begin{aligned}
\Delta^{1+2} z_{i j}= & \Delta_{x}\left(\Delta_{y y}^{2} z_{i j}\right)=\Delta_{x}\left(z_{i j+2}-2 z_{i j+1}+z_{i j}\right)= \\
= & \left(z_{i+1, y+2}-2 z_{i+1, j+1}+z_{i+1, j}\right)- \\
& -\left(z_{i, j+2}-2 z_{i, j+1}+z_{i, j}\right) .
\end{aligned}
$$

С использованием разности функций двух переменных $z=f(x, y)$ можно построить интерполяционный полином, аналогичный интерполяционному полиному Ньютона. Предположим, что $P(x, y)$ целый полином, где

$$
\Delta_{x}^{m+n} m_{y} n P\left(x_{0} y_{0}\right)=\Delta^{m+n} z_{00},
$$

где $m, n=0,1,2 \ldots$.

Разложение полинома $P(x, y)$ по обобщенным степеням разностей $x-x_{0}$ и $y-y_{0}$ запишется следующим образом:

$$
\begin{gathered}
P(x, y)=C_{00}+C_{10}\left(x-x_{0}\right)+C_{01}\left(y-y_{0}\right)+ \\
+C_{20}\left(x-x_{0}\right)\left(x-x_{1}\right)+C_{11}\left(x-x_{0}\right)\left(y-y_{0}\right)+ \\
+C_{02}\left(y-y_{0}\right)\left(y-y_{1}\right)+\ldots .
\end{gathered}
$$

Поскольку $x=x_{0}$ и $y-y_{0}$, то с учетом формулы (4) полином примет вид

$$
P\left(x_{0}, y_{0}\right)=z_{00}=C_{00} .
$$

Составив два полинома $P(x, y)$ конечных разностей первого порядка, получим уравнение

$$
\begin{gathered}
\Delta_{x} P(x, y)=C_{10} h+2 C_{20} h\left(x-x_{0}\right)+C_{11} h\left(y-y_{0}\right)+\ldots, \\
\Delta_{x} P(x, y)=C_{01} k+C_{11} k\left(x-x_{0}\right)+2 C_{02} k\left(y-y_{0}\right)+\ldots .
\end{gathered}
$$

С учетом того, что $x=x_{0}$ и $y=y_{0}$ и на основании формулы (4) получим

$$
\begin{aligned}
& \Delta_{x} P\left(x_{0}, y_{0}\right)=\Delta^{110} z_{00}=C_{10} h ; \\
& \Delta_{y} P\left(x_{0}, y_{0}\right)=\Delta^{0+1} z_{00}=C_{01} k,
\end{aligned}
$$

где $C_{10}=\frac{\Delta^{110} z_{00}}{h} ; C_{01}=\frac{\Delta^{0+1} z_{00}}{k} ;$ (9)

Найдем два полинома $P(x, y)$ конечных разностей второго порядка:

$$
\begin{aligned}
& \Delta_{x x} P(x, y)=2 ! C_{20} h^{2}+\ldots, \\
& \Delta_{x y} P(x, y)=C_{11} h k+\ldots, \\
& \Delta_{y y} P(x, y)=2 ! C_{02} k^{2}+\ldots .
\end{aligned} .
$$

При $x=x_{0}$ и $y=y_{0}$ полином второго порядка примет следующий вид:

$$
\begin{aligned}
& \Delta_{x x} P\left(x_{0}, y_{0}\right)=\Delta^{2+0} z_{00}=2 ! C_{20} h^{2} ; \\
& \Delta_{x y} P\left(x_{0}, y_{0}\right)=\Delta^{1+1} z_{00}=C_{11} h k ; \\
& \Delta_{y y} P\left(x_{0}, y_{0}\right)=\Delta^{0+2} z_{00}=2 ! C_{02} k^{2},
\end{aligned}
$$

где $C_{20}=\frac{1}{2 !} \frac{\Delta^{2+0} z_{00}}{h^{2}}, C_{11}=\frac{1}{2 !} \frac{\Delta^{1+1} z_{00}}{h k}, C_{02}=\frac{1}{2 !} \frac{\Delta^{0+2} z_{00}}{k^{2}}$.

2020

Аналогично вычисляем дальнейшие коэффициенты разложения выражения (5). Подставив найденные коэффициенты в формулу (5), получим 
интерполяционный полином для функции двух переменных:

$$
\begin{gathered}
P(x, y)=z_{00}+\left[\frac{\Delta^{1+0} z_{00}}{h}\left(x-x_{0}\right)+\frac{\Delta^{0+1} z_{00}}{k}\left(y-y_{0}\right)\right]+ \\
+\frac{1}{2 !}\left[\frac{\Delta^{2+0} z_{00}}{h^{2}} \times\left(x-x_{0}\right)^{2}+2 \frac{\Delta^{1+1} z_{00}}{h k}\left(x-x_{0}\right)\left(y-y_{0}\right)+\right. \\
\left.+\frac{\Delta^{0+2} z_{00}}{k^{2}}\left(y-y_{0}\right)^{2}\right]+\cdots .
\end{gathered}
$$

При интерполировании функции $f(x, y)$

$$
f(x, y) \approx P(x, y)
$$

для удобства вычислений введем переменные

$$
\frac{x-x_{0}}{h}=p ; \frac{y-y_{0}}{k}=q .
$$

В этом случае $\frac{x-x_{1}}{h}=p-1 ; \frac{y-y_{1}}{k}=q-1$ и т.д.

В результате принятых обозначений формула (12) примет следующий вид:

$$
\begin{aligned}
z \approx z_{00}+ & \left(p \Delta^{1+0} z_{00}+q \Delta^{0+1} z_{00}+\frac{1}{2 !}\left[p(p-1) \Delta^{2+0} z_{00}+\right.\right. \\
& \left.+2 p q \Delta^{1+1} z_{00}+q(q-1) \Delta^{0+2} z_{00}\right]+\ldots
\end{aligned}
$$

где $x=x_{0}+p h, y=y_{0}+q k$.

Если предположить, что $p=0$ или $q=0$, то формула 5 перейдет в соответствующую интерполяционную формулу Ньютона:

$$
\begin{aligned}
& f(x, y) \approx p(x, y) \approx K_{1}+K_{2}\left(x-x_{0}\right)+K_{3}\left(y-y_{0}\right)+ \\
& +K_{4}\left(x-x_{0}\right)^{2}+K_{5}\left(x-x_{0}\right)\left(y-y_{0}\right)+K_{6}\left(y-y_{0}\right)^{2} .
\end{aligned}
$$

Поверхность области номинала, устанавливающую функциональную связь номинала, между энергическим параметром, степенью загрузки и коэффициента вариации можно записать в виде уравнения:

$$
P(x, y)=K_{1}+K_{2 x}+K_{3 x}+K_{4 x^{2}}+K_{5 x y}+K_{6 y^{2}},
$$

или

$$
M(y)=a_{0}+a_{1} \lambda_{\bar{M}}+a_{2} v_{M}+a_{3} \lambda_{M}^{2}+a_{4} \lambda_{M} v_{M}+a_{5} v_{M}^{2} .
$$

Коэффициенты $K_{1}-K_{6}$ или $a_{0}-a_{5}$ определяются на ЭВМ по стандартным программам многофакторного регрессионного анализа.

Заключение. В результате проведения трех опытов вблизи номинала можно определить значения $\lambda_{\text {м }}{ }_{\text {м }}$ и $M(y)$. Подставив их в уравнение (18), получим уравнение поверхности представляющее собой аппроксимацию эксплуатационных точек математических ожиданий функциональных параметров с погрешностью не более $2 \%$. Оно позволит найти экстремумы параметров, необходимые при тестовом диагностировании или при определении максимальной производительности машинно-тракторного агрегата.

\section{СПИСОК ЛИТЕРАТУРЫ}

1. Савельев А.П. Диагностирование тракторов по динамическому состоянию машинно-тракторных агрегатов. - Саранск: Изд-во Мордов. ун-та, 1993.$220 \mathrm{c}$.

2. Глотов С.В. Оценка эффективности функционирования тракторов. - Саранск, 2003. - 188 с.

3. Савельев А.П., Калачин С.В., Шкрабак Р.В. Обоснование допустимых значений эксплуатационных параметров в системе «Оператор - машинно-тракторный агрегат» // Известия Санкт-Петербургского государственного аграрного университета. 2011. № 22. - С. 302-305.

4. Савельев А.П. Повышение достоверности диагностирования дизелей по функциональным параметрам // Сельский механизатор. - №2. - М., 2019. - С. 34.

Савельев Анатолий Петрович, $\partial-p$ техн. наук, проф., Национальный исследовательский Мордовский государственный университет им. Н.П. Огарева. Россия.

430005, Республика Мордовия, г. Саранск, ул. Большевистская, 68.

Тел.: 89272768168.

Шкрабак Владимир Степанович, $\partial-p$ техн. наук, проф. кафедры «Безопасность технологических процессов и производств», Санкт-Петербургский государственный аграрный университет. Россия.

196601, г. Санкт-Петербург - Пушкин, Петербургское mocce, 2.

Тел.: (812) 451-76-18.

Ключевые слова: функциональные параметры двигателей; экстремальные значения; интегрирование функций двух переменных; регрессионный анализ; аппроксимация.

\section{METHOD FOR DETERMINING EXTREME VALUES OF ENGINE FUNCTIONAL PARAMETERS BASED ON THE RESULTS OF EXPERIMENTAL STUDIES}

Saveliev Anatoly Petrovich, Doctor of Technical Sciences, Professor, National Research Mordovia State University named after N.P. Ogaryov. Russia.

Shkrabak Vladimir Stepanovich, Doctor of Technical Sciences, Professor of the chair "Safety of Technological Processes and Productions", St. Petersburg State Agrarian University. Russia.
Keywords: functional parameters of engines; extreme values; integration of functions of two variables; regression analysis; approximation.

A method for determining the extreme values of the functional parameters of engines in extreme studies of machine-tractor units by integrating the function of two variables is presented. 